\title{
Manifestaciones tomográficas de los subtipos del adenocarcinoma invasor de pulmón
}

\section{Tomographic manifestations of the subtypes of invasive adenocarcinoma of the lung}

Carmen I. Vargas-Díaz y Nelson Acuña-Aguilar ${ }^{2 *}$

${ }^{1}$ Departamento de Imagenología, Instituto Nacional de Enfermedades Respiratorias Ismael Cosío Villegas; ${ }^{2}$ Servicio de Tomografia Computarizada, Departamento de Radiología e Imagen, Instituto Nacional de Ciencias Médicas y Nutrición Salvador Zubirán. Ciudad de México, México

\section{RESUMEN}

Introducción: El cáncer de pulmón es causa de la mayor parte de defunciones en el mundo, y el adenocarcinoma es el tipo más frecuente. Se divide en lesiones preinvasivas y lesiones invasivas, con cinco subtipos: lepídico, acinar, papilar, micropapilar y sólido. La importancia de conocer su comportamiento radiológico es que nos acercaría a un diagnóstico de mejor precisión, teniendo en cuenta que la decisión terapéutica se basa en el subtipo histológico. Objetivo: Conocer las manifestaciones tomográficas de los diferentes subtipos histológicos del adenocarcinoma invasor de pulmón. Método: Estudio descriptivo y retrospectivo. Se revisaron el archivo de imágenes, el expediente clínico y los resultados de patología de 113 pacientes con diagnóstico de adenocarcinoma invasor de los años 2015 y 2016. Resultados: El patrón de consolidación se presentó en los subtipos acinar y lepídico; el patrón de masa, en el sólido, el papilar y el micropapilar. Todos tuvieron diseminación hematógena, linfática y mixta. El enfisema fue el principal hallazgo asociado; el lepídido se asoció a vidrio deslustrado. La exposición a cigarrillos fue del 100\% para el subtipo micropapilar. Conclusiones: Se identificó la tendencia de presentación tomográfica de los diferentes subtipos de adenocarcinoma de pulmón; conociendo el comportamiento podemos acercarnos aún más al diagnóstico.

Palabras clave: Cáncer de pulmón. Adenocarcinoma invasor. Tomografía. 


\section{ABSTRACT}

Introduction: Lung cancer is responsible for most deaths in the world, adenocarcinoma is the most common type. It is divided into pre-invasive lesions and invasive lesions with five subtypes: lepidic, acinar, papillary, micropapillary and solid. The importance of knowing its radiological behavior is that it would bring us closer to a more accurate diagnosis, taking into account that the therapeutic decision is based on the histological subtype. Objective: To know the tomographic manifestations of the different histological subtypes of invasive adenocarcinoma of the lung. Method: Descriptive, retrospective study. The image file, clinical file and pathology results of 113 patients with a diagnosis of invasive adenocarcinoma from 2015 and 2016 were reviewed. Results: The consolidation pattern was presented in the acinar and lepidic subtypes, the mass pattern for the solid, papillary, and micropapillary. All had hematogenous, lymphatic and mixed spread. Emphysema was the main associated finding, the lepidid was associated with ground glass. Exposure to cigarettes was $100 \%$ for the micropapillary subtype. Conclusions: The tendency of the tomographic presentation of the different subtypes of lung adenocarcinoma was identified, knowing the behavior we can get even closer to the diagnosis.

Keywords: Lung cancer. Invasive adenocarcinoma. Tomography.

\section{INTRODUCCIÓN}

El cáncer es la principal causa de muerte; en 2015 se le atribuyeron 8.8 millones de defunciones en el mundo, y el cáncer de pulmón se encuentra en el primer lugar con 1.69 millones ${ }^{1}$. Dentro de este, el adenocarcinoma pulmonar es el tipo histológico más frecuente en la mayoría de los países ${ }^{2}$. Uno de los grandes avances en su tratamiento en la última década es el concepto de medicina personalizada, según el cual las decisiones terapéuticas se basan en la histología específica y las características genéticas del tumor del paciente ${ }^{3}$.

En 2015 se elaboró la nueva clasificación del cáncer pulmonar con enfoque interdisciplinario. El principal cambio lo sufrió el adenocarcinoma, con la inclusión de nueva terminología y la clasificación en lesiones preinvasivas e invasivas con sus diferentes subtipos $^{4,5}$. Se ratifica el uso estandarizado de técnicas diagnósticas, como la inmunohistoquímica, la tomografía computarizada y la biopsia por abordaje mínimamente invasivo $^{6,7}$. La importancia del adenocarcinoma radica en el incremento de casos en personas jóvenes, del sexo femenino y sin antecedentes de hábito tabáquico.

El adenocarcinoma invasivo se clasifica en cinco subtipos ${ }^{3}$ : lepídico, acinar, papilar, micropapilar y sólido (Fig. 1). Se diferencia del mínimamente invasivo por la infiltración del estroma mayor de $5 \mathrm{~mm}$ (Fig. 1A). Sus manifestaciones radiológicas son muy variadas y tiene un espectro muy amplio que traduce las estructuras involucradas; estas pueden caracterizarse por un patrón de consolidación lobar o multisegmentaria, atelectasia, vidrio 


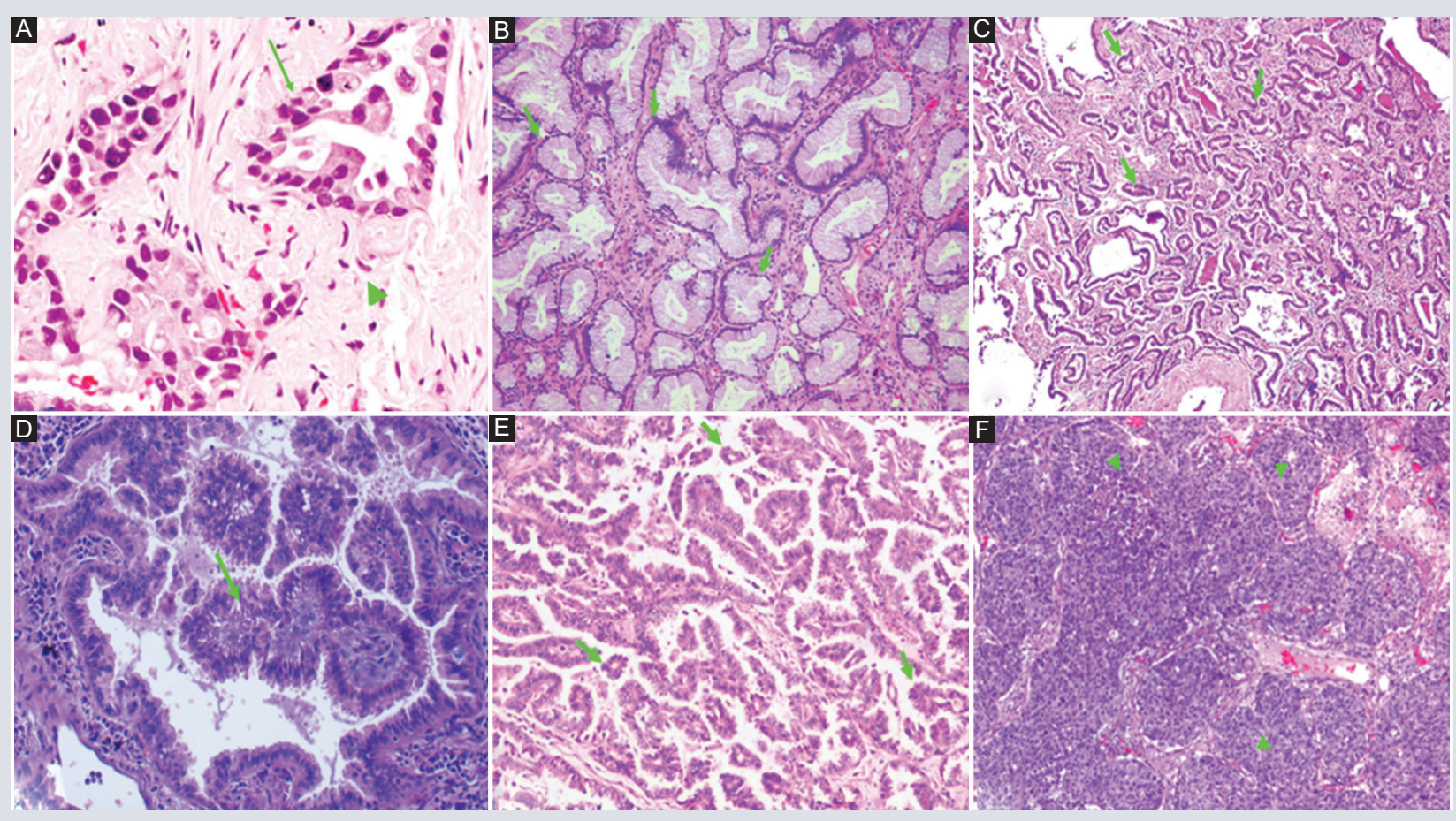

Figura 1. Fotomicrografías de adenocarcinoma de pulmón. A: seco fuerte 40x. Adenocarcinoma mínimamente invasivo (flecha) con invasión del estroma de $<5 \mathrm{~mm}$ (punta de flecha). B: seco débil 10x. Adenocarcinoma invasor subtipo lepídico (flechas). Los invasores presentan infiltración del estroma $>5 \mathrm{~mm}$. C: seco débil 10x. Adenocarcinoma invasor subtipo acinar (flechas) con arquitectura cribiforme. D: seco fuerte 40x. Adenocarcinoma invasor subtipo papilar (flecha) con centro fibrovascular. E: seco débil 10x.

Adenocarcinoma invasor subtipo micropapilar (flechas) sin centro fibrovascular. F: seco débil 10x. Adenocarcinoma invasor subtipo sólido (puntas de flecha) formando extensos mantos sólidos.

deslustrado, crazy paving o masa (Fig. 2). Puede tener diseminación hematógena manifestada por un patrón multinodular; la diseminación linfangítica se traduce por un patrón septal arrosariado asociado a engrosamiento del intersticio axial; o puede tener un patrón mixto hematógeno-linfático ${ }^{8-10}$ (Fig. 3A and $\mathrm{B}$ ). Otros hallazgos incluyen el derrame pleural masivo libre unilateral y la presencia de adenopatías mediastinales, hiliares e intrapulmonares (Fig. 3C and D). La diseminación extratorácica puede ser variable (Fig. 4).

El interés de conocer la tendencia de los diferentes patrones que se traducen por imagen permitiría su valoración oportuna, siendo indispensable describirlos por el valor de mal pronóstico que tienen los subtipos micropapilar y sólido ${ }^{11-15}$.

Existen múltiples estudios que abordan al adenocarcinoma de pulmón haciendo énfasis en la variedad preinvasiva para el diagnóstico precoz y mejorar la respuesta al tratamiento. Sin embargo, a la mayor parte de la población se le diagnostica la enfermedad cuando está en su variedad invasiva ${ }^{16-18}$.

El presente trabajo se centrará en describir la tendencia de los patrones tomográficos de los diferentes subtipos histológicos del adenocarcinoma de tipo invasivo, los cuales tienen 

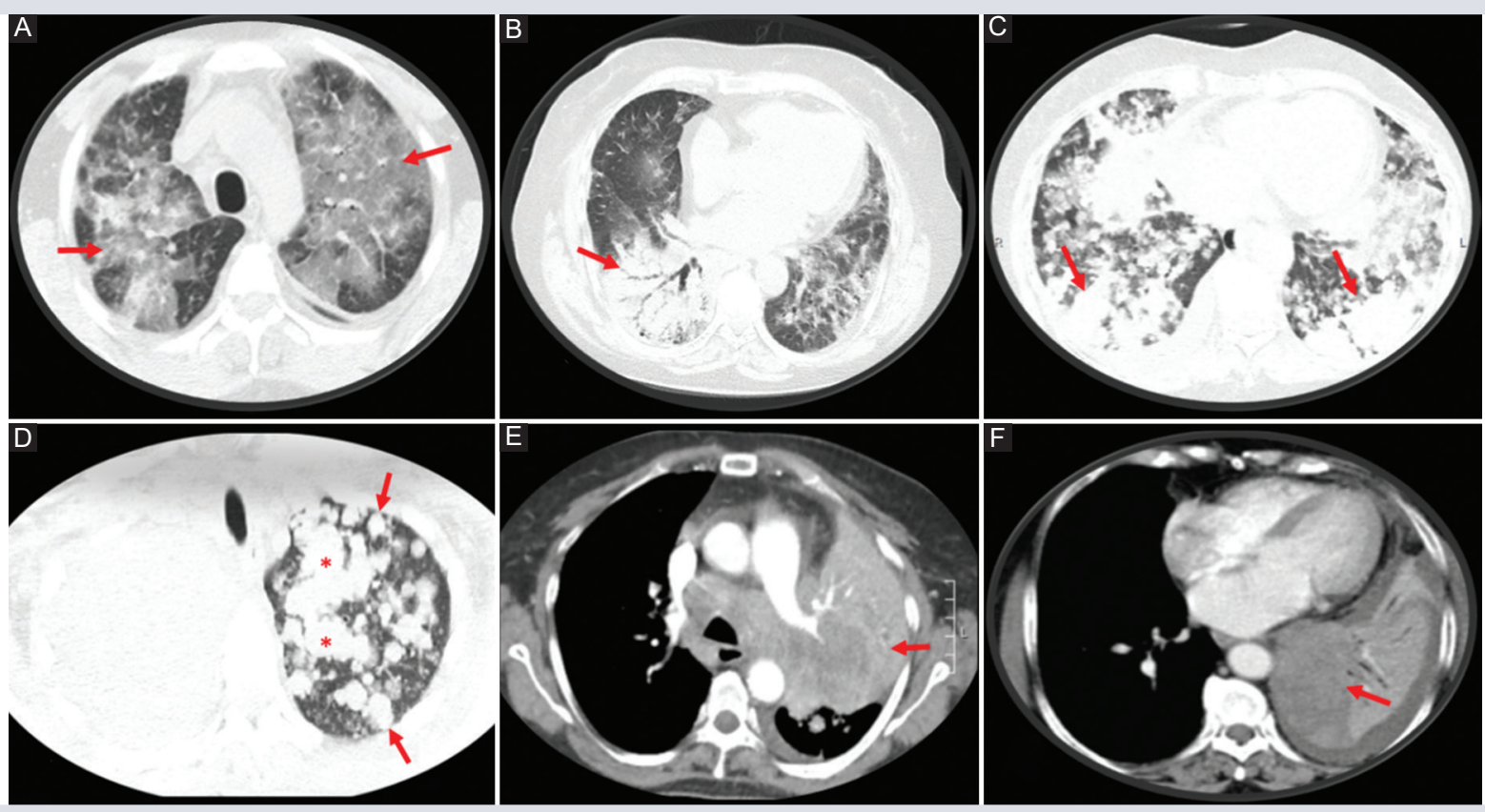

Figura 2. Manifestaciones tomográficas del adenocarcinoma de pulmón invasivo. A: tomografía computarizada (TC) simple de tórax en ventana pulmonar que muestra un patrón en vidrio deslustrado bilateral (flechas). B: TC simple de tórax en ventana pulmonar que muestra un patrón de consolidación lobar con broncograma aéreo (flecha). C: TC simple de tórax en ventana pulmonar que muestra un patrón de consolidación de distribución bilateral periférica con tendencia a la coalescencia (flechas). D: TC simple de tórax en ventana pulmonar que muestra un patrón multinodular (flechas) que confluye formando masas (asteriscos). E: TC contrastada de tórax en ventana de mediastino que muestra un patrón de atelectasia del lóbulo superior izquierdo (flecha). F: TC contrastada de tórax en ventana de mediastino que muestra un patrón de masa en el lóbulo inferior izquierdo (flecha).

valor pronóstico diferente para cada uno de ellos, además de indagar sobre los factores de exposición que pudieran tener los pacientes.

El objetivo de este estudio fue conocer la correlación de los hallazgos tomográficos que presentan los diferentes subtipos del adenocarcinoma invasivo, su relación con los antecedentes de exposición y el resultado de histopatología.

\section{MÉTODO}

Estudio observacional, descriptivo y retrospectivo. Se revisaron los expedientes clínicos electrónicos, el archivo de imágenes y los resultados de histopatología de los pacientes con diagnóstico de adenocarcinoma de pulmón del Instituto Nacional de Enfermedades Respiratorias Ismael Cosío Villegas, de la Ciudad de México, durante el periodo 2016-2017.

Las tomografías se realizaron con un equipo de la marca SIEMENS SOMATOM definition AS 128. De acuerdo con el protocolo, se adquieren cortes de $3 \mathrm{~mm}$, y según el caso se realizan reconstrucciones finas en ventana para mediastino y parénquima pulmonar, con contraste yodado no iónico intravenoso a dosis de $2 \mathrm{mg} / \mathrm{kg}$, a un caudal de $3 \mathrm{ml} / \mathrm{s}$, mediante inyector automático de doble cabezal. Los resultados de patología fueron evaluados nuevamente por un patólogo pulmonar. 


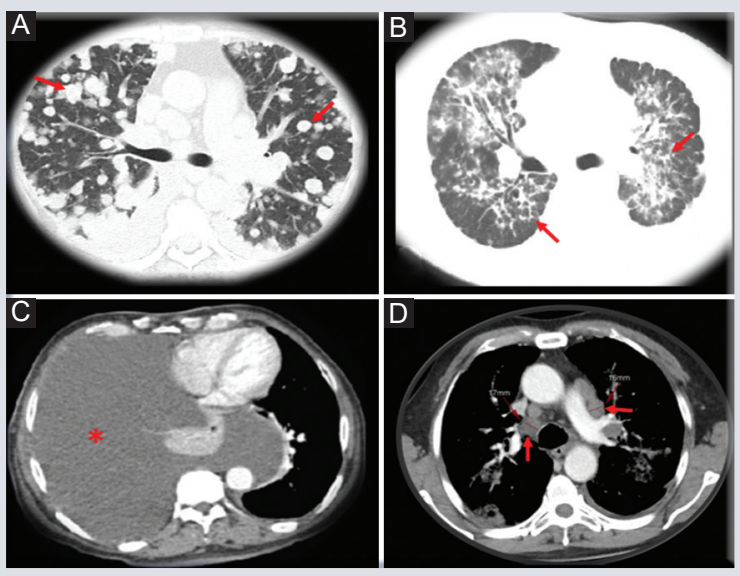

Figura 3. Tipos de diseminación torácica y hallazgos asociados del adenocarcinoma invasor de pulmón. A: tomografía computarizada (TC) simple de tórax en ventana pulmonar que muestra diseminación hematógena manifestada por un patrón nodular centrolobulillar (flechas). B: TC simple de tórax en ventana pulmonar que muestra diseminación linfática manifestada por el engrosamiento arrosariado del intersticio axial (flechas). C: TC simple de tórax en ventana de mediastino en la que se evidencia derrame pleural derecho masivo (asterisco) con desplazamiento del mediastino. D: TC simple de tórax en ventana de mediastino que muestra adenopatías mediastinales (flechas).

Todos los hallazgos se tabularon en una plantilla de recolección de datos elaborada con las variables del estudio; se utilizaron los paquetes estadísticos SPSS 11.4 y Microsoft Excel 2016.

\section{RESULTADOS}

Cumplieron los criterios 113 pacientes con diagnóstico de adenocarcinoma invasivo. De ellos, 57 fueron del sexo masculino y 56 del sexo femenino, y el rango de edad fue de 32 a 82 años, con una media de 60 años. Las frecuencias de distribución de los diferentes subtipos se muestran en la tabla 1. Las manifestaciones tomográficas de los subtipos de
Tabla 1. Frecuencia de presentación de los subtipos de adenocarcinoma de pulmón

\begin{tabular}{|l|c|}
\hline Subtipo & $\mathbf{n}$ \\
\hline Lepídico & 21 \\
\hline Acinar & 36 \\
\hline Papilar & 11 \\
\hline Micropapilar & 6 \\
\hline Sólido & 39 \\
\hline Total & 113 \\
\hline
\end{tabular}

adenocarcinoma fueron diversos (Tabla 2), existiendo predilección por algún tipo de presentación.

Los diferentes subtipos tuvieron los tres tipos de diseminación de la enfermedad: hematógena, linfática y mixta (Tabla 3). Existieron diferentes hallazgos asociados, siendo algunos más frecuentes en determinados subtipos (Tabla 4).

Los estadios de las lesiones fueron avanzados, encontrándose extensión torácica a la pleura, el pericardio, los vasos o alguna estructura mediastínica en un gran porcentaje de los casos: en el subtipo lepídico el $86 \%$, en el acinar el $50 \%$, en el papilar el $100 \%$, en el micropapilar el $100 \%$ y en el sólido el $100 \%$.

Se evidenciaron metástasis extratorácicas del adenocarcinoma invasor hacia el abdomen (glándula suprarrenal e hígado), el cerebro y los huesos: en el subtipo lepídico el $24 \%$, en el acinar el $42 \%$, en el papilar el $45 \%$, en el micropapilar el $100 \%$ y en el sólido el $67 \%$.

Los antecedentes clínicos de exposición a partículas estuvieron presentes en los pacientes con los diferentes subtipos de 


\section{C.I. Vargas-Díaz, N. Acuña-Aguilar: TC de adenocarcinoma invasor de pulmón}
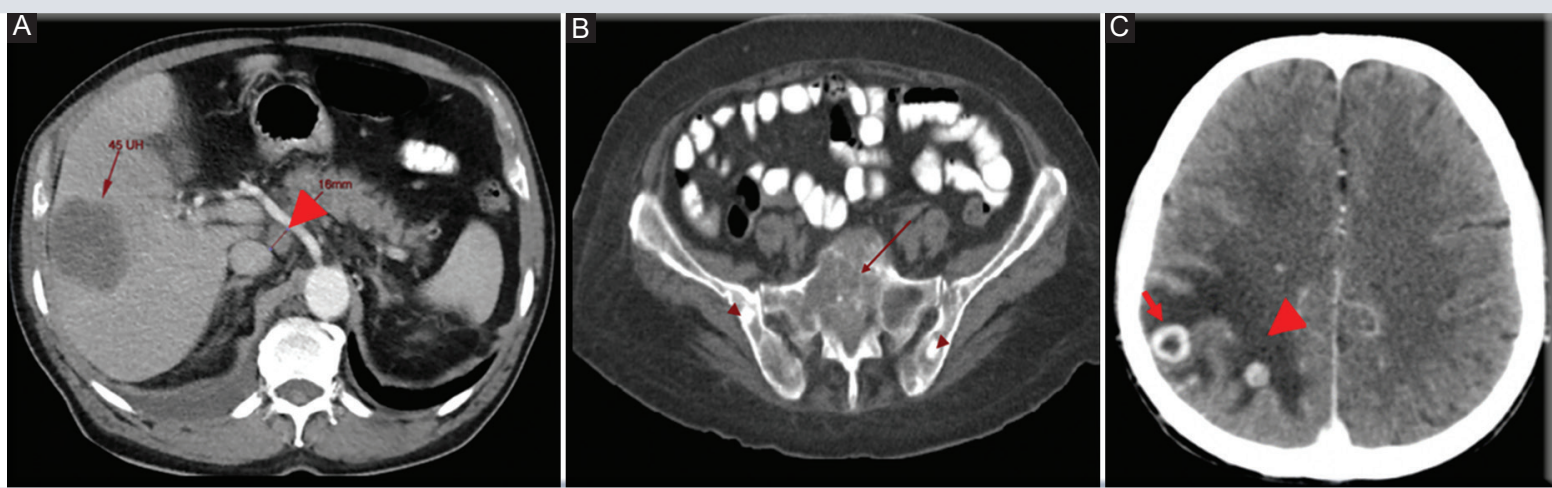

Figura 4. Tipos de metástasis extratorácicas del adenocarcinoma de pulmón invasivo. A: tomografía computarizada (TC) con contraste intravenoso y oral en ventana para tejidos blandos que muestra metástasis hepática (flecha) con mínimo reforzamiento al medio de contraste con atenuación de $45 \mathrm{UH}$. Se identifica una adenopatía celiaca de $16 \mathrm{~mm}$ (punta de flecha). B: TC simple de pelvis con contraste oral en ventana de tejidos blandos que evidencia en las primeras vertebras sacras metástasis líticas (flechas), y en el ilíaco metástasis blásticas (puntas de flecha). C: TC con contraste intravenoso de cerebro en la que se observa metástasis nodular en anillo (flecha) y edema vasogénico periférico (punta de flecha) en el lóbulo parietal derecho.

Tabla 2. Manifestaciones tomográficas de los subtipos de adenocarcinoma invasor de pulmón

\begin{tabular}{|l|c|c|c|c|c|}
\hline & Lepídico & Acinar & Papilar & Micropapilar & Sólido \\
\hline Consolidación & 16 & 25 & 3 & 0 & 1 \\
\hline Atelectasia & 0 & 3 & 0 & 0 & 2 \\
\hline Vidrio deslustrado & 2 & 2 & 1 & 0 & 0 \\
\hline Crazy paving & 1 & 1 & 0 & 3 & 30 \\
\hline Masa & 2 & 5 & 6 & 2 & 2 \\
\hline Multinodular & 0 & 0 & 1 & 6 & 39 \\
\hline Total & 21 & 36 & 11 & \\
\hline
\end{tabular}

Tabla 3. Patrón de diseminación de los subtipos de adenocarcinoma invasor de pulmón

\begin{tabular}{|l|c|c|c|c|c|}
\hline & Lepídico & Acinar & Papilar & Micropapilar & Sólido \\
\hline Hematógeno/multinodular & 4 & 11 & 3 & 2 & 11 \\
\hline Linfangítico reticular/nodular & 6 & 2 & 0 & 0 & 6 \\
\hline Mixto & 5 & 14 & 3 & 4 & 8 \\
\hline
\end{tabular}

adenocarcinoma (Tabla 5). La exposición a cigarrillos fue del 100\% para el micropapilar, del $64 \%$ para el papilar y del $49 \%$ para el sólido. La exposición a humo de leña fue del $43 \%$ para el subtipo lepídico y del 39\% para el acinar. 
Tabla 4. Hallazgos asociados de los subtipos de adenocarcinoma invasor de pulmón

\begin{tabular}{|l|c|c|c|c|c|}
\hline & Lepídico & Acinar & Papilar & Micropapilar & Sólido \\
\hline Derrame pleural masivo & 1 & 6 & 1 & 0 & 1 \\
\hline Derrame pleural leve & 12 & 18 & 5 & 11 & 6 \\
\hline Adenopatías & 20 & 29 & 0 & 0 & 36 \\
\hline Vidrio deslustrado & 16 & 9 & 3 & 2 & 12 \\
\hline Enfisema & 4 & 13 & 1 & 1 & 8 \\
\hline Otros & 5 & 5 & & \\
\hline
\end{tabular}

Tabla 5. Exposición a partículas de los pacientes con adenocarcinoma invasor de pulmón

\begin{tabular}{|l|c|c|c|}
\hline Subtipo & $\begin{array}{c}\text { Partículas } \\
\text { inorgánicas }\end{array}$ & $\begin{array}{c}\text { Humo de } \\
\text { leña }\end{array}$ & Tabaco \\
\hline Lepídico & 4 & 9 & 4 \\
\hline Acinar & 5 & 14 & 11 \\
\hline Papilar & 3 & 0 & 7 \\
\hline Micropapilar & 2 & 0 & 6 \\
\hline Sólido & 9 & 1 & 19 \\
\hline
\end{tabular}

\section{DISCUSIÓN}

Según los resultados, el adenocarcinoma invasor de pulmón afecta por igual a hombres y mujeres, con una media de edad de 60 años. Los pacientes del subtipo sólido fueron los más jóvenes al diagnóstico, con una media de 58 años, y los de mayor edad fueron los del subtipo micropapilar, con una media de 69 años. Los pacientes de menor edad se encuentran en el subtipo papilar, con 32 años. El subtipo lepídico afectó más al sexo femenino, con un $62 \%$, no habiendo diferencias en los otros subtipos. Se encontró que el subtipo más frecuente de presentación es el sólido, con un 34\%, seguido del acinar con un $32 \%$, el lepídico con un $18 \%$, el papilar con un $10 \%$ y por último el micropapilar con un $5 \%$.

Se observó inclinación al patrón tomográfico de consolidación y de masa. El subtipo lepídico $(76 \%)$ y el acinar (69\%) presentaron tendencia al patrón de consolidación. El papilar (55\%), el micropapilar (50\%) y el sólido (77\%) presentaron predominio del patrón en masa.

El derrame pleural y las adenopatías se asociaron a todos los subtipos, debido al estadio avanzado de la enfermedad. Existe asociación del 38\% para el vidrio deslustrado en el lepídico y del 36\% para el enfisema en el acinar, y del $31 \%$ en el sólido; estos dos últimos probablemente relacionados con el consumo de tabaco.

No hubo diferencias significativas en cuanto al patrón de diseminación pulmonar por vía hematógena, linfática o mixta entre los diferentes subtipos de adenocarcinoma.

El humo de leña fue el factor de exposición más frecuente en el lepídico $(43 \%)$ y en el acinar (39\%). En el papilar (64\%), el micropapilar $(100 \%)$ y el sólido (49\%) hubo mayor exposición a los cigarrillos. Si bien los datos son 
solo significativos para el micropapilar y el papilar, se requieren más estudios para corroborar los resultados.

La extensión torácica al mediastino es más frecuente en los subtipos sólido y papilar, que estaría relacionada con su mayor agresividad.

En cuanto a la extensión extratorácica, se encontró cerebral, ósea, suprarrenal y hacia los ganglios retroperitoneales.

Los resultados presentados sobre las manifestaciones tomográficas del adenocarcinoma invasor de pulmón son inéditos, no encontrándose referencias bibliográficas, y tienen su importancia porque pueden servir en lugares donde no se cuenta con métodos de histopatología avanzados y a veces solo se dispone de la imagen para hacer el diagnóstico.

Conocer el comportamiento por la imagen de los subtipos de adenocarcinoma invasor servirá para aproximarse al diagnóstico en lugares donde no se puede acceder a métodos de patología avanzados. Como se evidenció en nuestra población, algunos subtipos se comportan de manera diferente en comparación con otro tipo de poblaciones.

\section{FINANCIAMIENTO}

Los autores declaran no haber recibido financiamiento alguno para realizar este trabajo.

\section{CONFLICTO DE INTERESES}

Los autores declaran que no existe conflicto de intereses.

\section{Responsabilidades éticas}

Protección de personas y animales. Los autores declaran que para esta investigación no se han realizado experimentos en seres humanos ni en animales.

Confidencialidad de los datos. Los autores declaran que en este artículo no aparecen datos de pacientes.

\section{Derecho a la privacidad y consentimiento} informado. Los autores han obtenido el consentimiento informado de los pacientes $\mathrm{y} / \mathrm{o}$ sujetos referidos en el artículo. Este documento obra en poder del autor de correspondencia.

\section{BIBLIOGRAFÍA}

1. Organización Mundial de la Salud. Nota descriptiva. Febrero de 2017. (Citado 30/09/2017). Disponible en: http://www.who.int/mediacentre/ factsheets/fs297/es

2. Lee HJ, Kim YT, Kang CH, Zhao B, Tan Y, Schwatz LH, et al. Epidermal growth factor receptor mutation in lung adenocarcinomas: relationship with CT characteristics and histologic subtypes. Radiology. 2013;268:254-64.

3. Travis WD, Brambilla E, Nicholson AG, Yatabe Y, Austin JH, Beasley MB, et al. The 2015 World Health Organization Classification of Lung Tumors. Impact of Genetic, Clinical and Radiologic Advances Since the 2004 Classification. J Thorac Oncol. 2015;10:1243-60.

4. Travis WD, Brambilla E, Muller-Hermelink HK, Harris CC. Pathology and genetics. En: Tumors of the lung, pleura, thymus and heart. Lyon, France: IARC Press; 2004.

5. Travis WD, Colby TV, Corrin B, Shimosato Y, Brambilla E. Histological typing of lung and pleural tumors. In: World Health Organization international histological classification of tumors. $3^{\text {rd }}$ ed. Berlin, Germany: Springer; 1999.

6. Borczuk AC, Shah L, Pearson GDP, Walter KL, Wang L, Austin JH, et al. Molecular signatures in biopsy specimens of lung cancer. Am J Respir Crit Care Med. 2004;170:167-74.

7. Solomon SB, Zakowski MF, Pao W, Thornton RH, Ladanyi M, Kris MG, et al. Core needle lung biopsy specimens: adequacy for EGFR and KRAS mutational analysis. AJR Am J Roentgenol. 2010;194:266-9.

8. Yoshizawa A, Motoi N, Riely GJ, Sima CS, Gerald WL, Kris MG, et al. Impact of proposed IASLC/ATS/ERS classification of lung adenocarcinoma: prognostic subgroups and implications for further revision of staging based on analysis of 514 stage I cases. Mod Pathol. 2011;24:653-64.

9. Sica G, Yoshizawa A, Sima CS, Azzoli CG, Downey RJ, Rusch VW, et al. A grading system of lung adenocarcinomas based on histologic pattern is predictive of disease recurrence in stage I tumors. Am J Surg Pathol. 2010;34:1155-62. 
10. Nagano T, Ishii G, Nagai K, Ito T, Kawase A, Takahashi K, et al. Structural and biological properties of a papillary component generating a micropapillary component in lung adenocarcinoma. Lung Cancer. 2010;67:282-9.

11. Ikeda K, Awai K, Mori T, Kawanaka K, Yamashita Y, Nomori H. Differential diagnosis of ground-glass opacity nodules: CT number analysis by threedimensional computerized quantification. Chest. 2007;132:984-90.

12. Godoy MC, Naidich DP. Subsolid pulmonary nodules and the spectrum of peripheral adenocarcinomas of the lung: recommended interim guidelines for assessment and management. Radiology. 2009;253:606-22.

13. Kim HY, Shim YM, Lee KS, Han J, Yi CA, Kim YK. Persistent pulmonary nodular ground-glass opacity at thin-section CT: histopathologic comparisons. Radiology. 2007;245:267-75.

14. Lee HJ, Goo JM, Lee CH, Park CM, Kim KG, Park EA, et al. Predictive CT findings of malignancy in ground-glass nodules on thin-section chest CT: the effects on radiologist performance. Eur Radiol. 2009;19:552-60.
15. Travis WD, Garg K, Franklin WA, Wistuba II, Sabloff B, Noguchi M, et al Evolving concepts in the pathology and computed tomography imaging of lung adenocarcinoma and bronchioloalveolar carcinoma. J Clin Oncol. 2005;23:3279-87.

16. Borczuk AC, Qian F, Kazeros A, Eleazar J, Assaad A, Sonett JR, et al. Invasive size is an independent predictor of survival in pulmonary adenocarcinoma. Am J Surg Pathol. 2009;33:462-9.

17. Sawada E, Nambu A, Motosugi U, Araki T, Kato S, Sato Y, et al. Localized mucinous bronchioloalveolar carcinoma of the lung: thin-section computed tomography and fluorodeoxyglucose positron emission tomography findings. Jpn J Radiol. 2010;28:251-8.

18. Akira M, Atagi S, Kawahara M, Iuchi K, Johkoh T. High-resolution CT findings of diffuse bronchioloalveolar carcinoma in 38 patients. AJR Am J Roentgenol. 1999;173:1623-9. 